\title{
Quality of life after pulmonary embolism: validation of the French version of the PEmb-QoL questionnaire
}

\author{
Mathilde Rochat ${ }^{*}$, Marie Méan², Andreas Limacher ${ }^{3}$, Olivier Hugli ${ }^{4}$, Frederikus A Klok ${ }^{5}$, Danny M Cohn ${ }^{6}$ \\ and Drahomir Aujesky ${ }^{2}$
}

\begin{abstract}
Background: The PEmb-QoL is a validated 40-item questionnaire to quantify health-related quality of life in patients having experienced pulmonary embolism (PE). It covers six health dimensions: frequency of complaints, activities of daily living limitations, work-related problems, social limitations, intensity of complaints, and emotional complaints. Originally developed in Dutch and English, we sought to prospectively validate the psychometric properties of a French version of the PEmb-QoL.

Methods: We performed a forward and backward translation of the English version of the PEmb-QoL into French. French-speaking consecutive adult patients with an acute, objectively confirmed PE admitted to the emergency department of a Swiss university hospital between 08/2009 and 09/2011 were recruited telephonically. We used standard psychometric tests and criteria to evaluate the acceptability, reliability, and validity of the French version of the PEmb-QoL. We also performed an exploratory factor analysis.

Results: Overall, 102 patients were enrolled in the study. The French version of the PEmb-QoL showed good reliability (internal consistency, item-total and inter-item correlations), reproducibility (test-retest reliability), and validity (convergent, discriminant) in French-speaking patients with PE. The exploratory factor analysis suggested three underlying dimensions: limitations in daily activity (items 4b-m, 5a-d), symptoms (items 1a-h and 7), and emotional complaints (items 9a-f and j).

Conclusion: We successfully validated the French version of the PEmb-QoL questionnaire in patients with PE. Our results show that the PEmb-QoL is a valuable tool for assessing health-related quality of life after PE in French-speaking patients.
\end{abstract}

\section{Introduction}

Acute venous thromboembolism (VTE), defined as deep vein thrombosis (DVT) and/or pulmonary embolism (PE), is common and has a high impact on morbidity, mortality, and costs of care [1,2]. Besides the transient discomfort related to acute VTE, health-related quality of life is substantially influenced by the development of VTE-related complications [3]. The long-term natural course in patients surviving an acute VTE event can be complicated by recurrent episodes of VTE, bleeding complications caused by anticoagulation treatment, the post-thrombotic

\footnotetext{
* Correspondence: Mathilde.Rochat@chuv.ch

${ }^{1}$ Department of Internal Medicine, Lausanne University Hospital, CHUV, Rue du Bugnon 46, 1011 Lausanne, Switzerland

Full list of author information is available at the end of the article
}

syndrome and in rare cases, chronic thromboembolic pulmonary hypertension $[4,5]$.

Disease-specific quality of life questionnaires are necessary to better detect treatment effects and change over time in patients having the same disease. While instruments to measure disease-specific quality of life exist for patients with DVT [6], the Pulmonary Embolism Quality of Life (PEmb-QoL) questionnaire was only recently developed to specifically address health-related quality of life in patients having experienced PE $[7,8]$.

The PEmb-QoL, originally developed in Dutch and translated into English, is a 40-item questionnaire that 
measures the impact of PE on quality of life from the patient's perspective over the past four weeks $[7,8]$. A study using the Dutch version of the PEmb-QoL found that patients with PE had an impaired quality of life compared to the age-matched general population [9]. Recently, a Norwegian version of the PEmb-QoL was successfully validated [10]. Given that the PEmb-QoL questionnaire is the only available validated instrument to assess QoL after PE [8], we aimed to prospectively validate the psychometric properties of a French version of the PEmb-QoL questionnaire (Additional file 1).

\section{Methods}

\section{PEmb-QoL questionnaire}

The PEmb-QoL questionnaire contains nine questions (40 items) covering six dimensions: frequency of complaints (Q1, 8 items), activities of daily living limitations (Q4, 13 items), work-related problems (Q5, 4 items), social limitations (Q6, 1 item), intensity of complaints (Q7 and Q8, 1 item each), and emotional complaints (Q9, 10 items). Responses are rated on a Likert response scale.

Because no French version of this questionnaire is available, we performed a forward-backward translation from the English version of the PEmb-QoL questionnaire into French according to previous published recommendations [11]. In a first step, two independent native French speakers, of whom one was a naïve translator without medical background, performed a forward translation from the original English version into French. In a second step, two naïve English speakers performed a backward translation into English. A committee of three experts reviewed all translations and reached a consensus on any discrepancy. The final French version is shown in the supplemental online appendix.

\section{Scoring the PEmb-QoL questionnaire}

The scales of Q1, Q4, Q5, and Q9 were reversed, with a low point score indicating a better quality of life. Two questions (Q2 'At what time of day are your lung symptoms most intense?' and Q3 'Compared to one year ago, how would you rate the condition of your lungs in general now?') were not scored. Item 4 a was considered missing if the answer was 'I do not work'. As described in the initial publication [8], the PEmb-QoL dimension scores were calculated by taking the mean of the constituting items. Dimension scores were then transformed to a scale from 0-100 to make them comparable across dimensions, with higher scores indicating worse outcome. To estimate the overall impact of PE on quality of life, we developed a PEmb-QoL summary score. In a first step, we transformed all item scores to a scale ranging from 0 to 100 . In a second step, we averaged these transformed scores (except items Q2 and Q3) to obtain an overall summary score.

\section{Study subjects}

We identified consecutive patients aged $>18$ years with an acute, objectively confirmed PE admitted at the emergency department of the Lausanne university hospital, Switzerland, from August 1, 2009 to September 30, 2011 using the hospital's electronic patient tracking system. The confirmation of PE was based on either a highprobability ventilation-perfusion lung scan or a positive computed tomography scan $[12,13]$. We telephonically invited all screened patients who survived the PE episode for study participation. Exclusion criteria were refusal to participate, insufficient spoken language ability in French, history of dementia based on chart review, and residence in a nursing home or outside Switzerland.

We chose a sample size of 100 patients to validate the French version of the PEmb-QoL questionnaire, which is in accordance with methodological recommendations $[14,15]$ and a previous similar validation study [16]. The local ethics committee (Commission cantonale (VD) d'éthique de la recherche sur l'être humain) approved the study and all patients provided written consent.

\section{Data collection}

Eligible, consenting patients received a baseline PEmbQoL and a French language version of the Short-Form Health Survey (SF-36) questionnaire per mail [17]. The SF-36 questionnaire is a well validated generic quality of life measure consisting of 36 items grouped into eight dimensions (physical functioning, social functioning, physical role functioning, emotional role functioning, mental health, vitality, bodily pain, and general health). The scores vary from 0 to 100 for each dimension, with higher values indicating better health $[18,19]$. The SF-36 also provides a physical and mental health summary score. Standardized dimension and summary scores of the SF-36 questionnaire were calculated using the U.S. 1998 reference population [20].

Patients were asked to complete and return both questionnaires using a pre-stamped return envelope. Participants were then mailed a second PEmb-QoL and SF-36 questionnaire ten days after the baseline evaluation. In case a patient returned an incomplete questionnaire, a study collaborator contacted the patient by telephone to complete all missing items.

We used patient medical records to collect the following baseline characteristics for all enrolled patients: age, gender, cardiopulmonary comorbidity (defined as any cardiac disease with systolic or diastolic ventricular dysfunction or any obstructive or restrictive pulmonary disease), active cancer (defined as cancer with ongoing oncologic or palliative treatment within the previous six months), obesity (defined as body mass index more than $30 \mathrm{~kg} / \mathrm{m}^{2}$ ), history of prior VTE, and the time interval between the index PE and study inclusion. 


\section{Psychometric evaluation of the French version of the PEmb-QoL questionnaire}

We used standard statistical tests and criteria to evaluate the acceptability, reliability, and validity of the PEmb-QoL $[6,14,16]$. Baseline characteristics were shown as proportions or medians and ranges, as appropriate. The transformed PEmb-QoL dimension scores were depicted in a box plot as medians with interquartile range (IQR).

Because acceptability affects the quality of the data obtained, it was assessed by examining completeness of data and score distribution. To examine floor and ceiling effects, we calculated the proportion of patients who achieved the lowest or highest possible score per dimension and in the overall PEmb-QoL summary score. Criteria for acceptability included $<15 \%$ floor and ceiling effects for dimensions and summary score [14].

Factor analysis is widely used to evaluate whether questionnaire items can be grouped into clusters representing different dimensions of the construct under study [21]. Because the factor analysis in the validation study by Klok et al. [8] did not explore the appropriate number of latent factors (underlying dimensions) but rather sought to confirm the pre-specified structure of the questionnaire based on six dimensions, we explored the number of underlying dimensions and grouping of items in an exploratory factor analysis. We used the principal factor method to analyze the correlation matrix and applied an orthogonal varimax rotation on the loading matrix. The number of retained factors was determined by a scree test [22], which suggested three latent factors with eigenvalues of 17.0, 3.9, and 1.7. After rotation, the three factors accounted for $35 \%, 22 \%$, and $19 \%$ of the total variance, respectively. Question 4a ('Do your lung symptoms now limit you in daily activities at work?') was omitted from the factor analysis because almost half of patients in our sample did not work. An item was considered to load on a given factor if the loading was $>0.3$ for this factor.

We assessed reliability by determining internal consistency, which was measured by Cronbach's alpha, average inter-item correlation, item-total correlation, and the association between dimensions of the PEmb-QoL scores using pairwise Spearman correlation coefficients. Internal consistency refers to the extent to which items comprising the score measure the same construct (i.e., homogeneity of the score), and was considered acceptable when Cronbach's alpha was between 0.7 and 0.95 [14]. We regarded an item-total correlation $>0.2$ and an average inter-item correlation $>0.3$ as good [23].

We tested reproducibility by repeating the PEmb-QoL questionnaire after ten days (test-retest reliability). Testretest reliability measures the degree to which repeated measurements applied to the same individuals provide similar answers. We decided that a time period of ten days between the repeated distributions of the questionnaires was long enough to prevent recall bias but short enough to ensure that a clinical change in the symptoms being measured was unlikely to occur. Test-retest reliability was expressed as an intra-class correlation coefficient, with values $>0.7$ indicating good test-retest reliability [14].

Construct validity refers to the extent to which PEmbQoL scores relate to other measures in a manner consistent with theoretically derived hypotheses [14]. We first assessed construct validity by calculating pairwise Spearman correlation coefficients between PEmb-QoL and SF36 dimension and summary scores (convergent validity), as done in previous studies $[7,8]$. Because both questionnaires were developed to assess health-related quality of life, we assumed that the two measures would be correlated in a moderate range, one being a diseasespecific and the other a generic health-related quality of life questionnaire.

We assessed discriminant validity by examining whether PEmb-QoL scores were correlated with measures of unrelated constructs, i.e. patient age, sex, and clinical characteristics (obesity, cancer, and cardiopulmonary diseases). A similar approach was used in previous studies examining discriminant validity of a similarly structured quality of life questionnaire for DVT $[6,16]$. Our hypothesis was that correlation with age, gender, and clinical characteristics would be weak.

\section{Results}

\section{Patient sample}

Of 242 patients with PE screened, 61 could not be reached, 42 refused to participate, 20 had dementia, 8 lived in a nursing home, 6 were unable to speak French, and 3 lived abroad, leaving a final study sample of 102 patients. Overall, 46 patients (45\%) were aged $\geq 65$ years, and $39 \%$ were women (Table 1). The median (range) time between occurrence of the index PE and study enrollment was 15 (5-23) months.

Table 1 Baseline patient characteristics $(n=102)$

\begin{tabular}{ll}
\hline Characteristic & $\mathbf{n}(\%)$ or median (range) \\
\hline Age, years & $63(26-93)$ \\
Female gender & $40(39)$ \\
Obesity* & $13(13)$ \\
Active cancer & $10(10)$ \\
Cardiopulmonary comorbidity ${ }^{\dagger}$ & $13(13)$ \\
History of venous thromboembolism & $18(18)$ \\
Central pulmonary embolism & $10(10)$ \\
Time since pulmonary embolism (months) & $15(5-23)$
\end{tabular}

*Body mass index $>30 \mathrm{~kg} / \mathrm{m}^{2}$.

${ }^{\dagger}$ Any cardiac disease with systolic or diastolic ventricular dysfunction or any obstructive or restrictive pulmonary disease. 
Psychometric characteristics of the French version of the PEmb-QoL questionnaire

\section{Acceptability}

All questionnaires were returned by participants (response rate $100 \%)$. We contacted 33 participants (32\%) by phone to complete missing items. Overall, 41 participants (40\%) answered 'I do not work' for the question Q4a.

The median PEmb-QoL dimension and overall summary scores are shown in Figure 1. All dimensions had floor effects, ranging from $13 \%$ for emotional complaints (Q9) to 66\% for social limitations (Q6) (Table 2). Ceiling effects were $\leq 2 \%$ for all dimensions except for workrelated problems $(18 \%)$.

\section{Factor analysis}

We explored the number of underlying dimensions and grouping of items in an exploratory factor analysis, which suggested three latent factors (dimensions): limitations in daily activity (items 4b-m, 5a-d), symptoms (items 1a-h and 7), and emotional complaints (items 9a-f and j) (Table 3). Three items were not clearly assignable to a meaningful dimension in the French version of the PEmb-QoL: item 6 ("During the past four weeks, to what extend have your lung symptoms interfered with your normal social activities with family, friends, neighbors, or groups?"), item 8 ("How much breathlessness have you experienced in the past four weeks?") and item $9 \mathrm{~g}$-i ("How much of the time during the past four weeks ( $\mathrm{g}$ ) did you feel that you were a burden to your family and friends, (h)

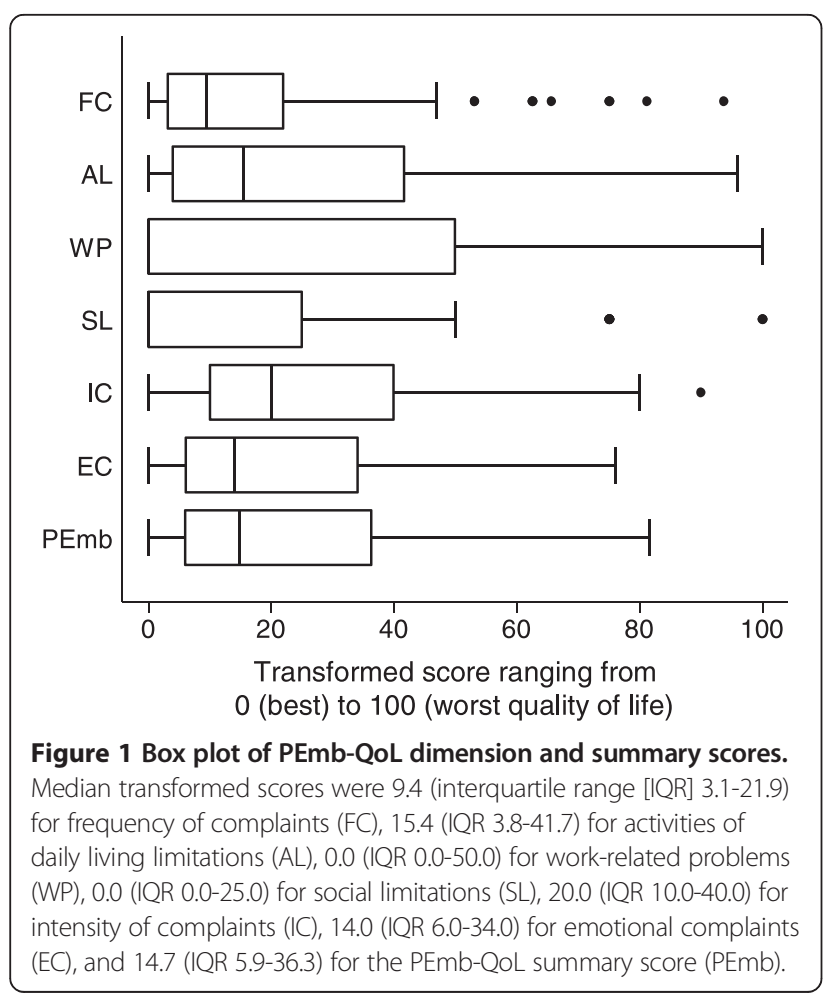

Table 2 Floor and ceiling effects of the PEmb-QoL dimension and summary scores

\begin{tabular}{|c|c|c|}
\hline & $\begin{array}{l}\text { Floor effect* } \\
\text { Percent }\end{array}$ & Ceiling effect $^{\dagger}$ \\
\hline Frequency of complaints (Q 1) & 25 & 0 \\
\hline Activities of daily living limitations (Q 4) & 20 & 0 \\
\hline Work-related problems (Q 5) & 60 & 18 \\
\hline Social limitations (Q 6) & 66 & 2 \\
\hline Intensity of complaints (Q 7 and Q 8) & 20 & 0 \\
\hline Emotional complaints (Q 9) & 13 & 0 \\
\hline PEmb-QoL summary score & 3 & 0 \\
\hline
\end{tabular}

were you afraid to exert yourself, (i) did you feel limited in taking a trip?").

\section{Reliability and reproducibility}

Almost all Cronbach's alpha coefficients were $>0.9$ except one (intensity of complaints, 0.7), indicating high internal consistency (Table 4). Items were positively correlated with each other, with all average inter-item correlations $>0.3$. All item-total correlation values were $>0.2$, ranging from 0.53 to 0.92 (data not shown for individual items). PEmb-QoL dimensions were moderately well correlated between themselves $(0.53 \leq \mathrm{r} \leq 0.83)$, with the highest correlation being between intensity of complaints and frequency of complaints $(r=0.83)$ and between intensity of complaints and emotional complaints $(r=0.75)$. Intra-class correlation coefficients for the testretest analysis were high, ranging between 0.85 for social limitations and 0.96 for emotional complaints (Table 5).

\section{Construct validity (convergent, discriminant)}

We did a correlation analysis using SF-36 component scores to assess convergent validity of the PEmb-QoL dimension and overall summary scores. The PEmb-QoL dimensions activities of daily living limitations, work-related problems, social limitations, and intensity of complaints showed higher correlations with the SF-36 Physical Component Summary, whereas frequency of complaints and emotional complaints had higher correlations with the SF36 Mental Component Summary (Table 6). Overall, these correlations supported a good convergent validity. The PEmb-QoL dimension and overall summary scores were only weakly correlated with clinical characteristics, indicating a good discriminant validity (Table 7).

\section{Discussion}

In our validation study, the PEmb-QoL questionnaire showed not only a high internal consistency and interitem and item-total correlation but also high test-retest 
Table 3 Factor analysis of the PEmb-QoL questionnaire*

\begin{tabular}{|c|c|c|c|c|}
\hline \multirow{2}{*}{ Item } & & Factor 1 & Factor 2 & Factor 3 \\
\hline & & \multicolumn{3}{|c|}{ Factor loadings $^{\dagger}$} \\
\hline $1 \mathrm{a}$ & Pain behind or between the shoulder blades? & 0.1031 & 0.6572 & 0.0632 \\
\hline $1 b$ & Pain on or in the chest? & 0.0770 & 0.7807 & 0.2031 \\
\hline $1 \mathrm{c}$ & Pain in the back? & 0.2416 & 0.6543 & 0.1467 \\
\hline $1 d$ & Sensation of pressure? & 0.2262 & 0.6425 & 0.2495 \\
\hline 1e & Feeling that there is "still something there"? & 0.2990 & 0.6354 & 0.2826 \\
\hline 1f & "Burning sensation" in the lung? & 0.09300 & 0.7339 & 0.2218 \\
\hline $1 \mathrm{~g}$ & "Nagging feeling"? & 0.1382 & 0.7831 & 0.2318 \\
\hline $1 \mathrm{~h}$ & Difficulty in breathing or breathlessness? & 0.4637 & 0.5088 & 0.3665 \\
\hline $4 b$ & Daily activities at home & 0.7796 & 0.3230 & 0.2529 \\
\hline $4 c$ & Social activities & 0.7123 & 0.1880 & 0.2811 \\
\hline $4 d$ & Vigorous activities & 0.7471 & 0.2588 & 0.1131 \\
\hline $4 e$ & Moderate activities & 0.8412 & 0.1479 & 0.1001 \\
\hline $4 f$ & Lifting or carrying activities & 0.8319 & 0.1576 & 0.1946 \\
\hline $4 \mathrm{~g}$ & Climbing several flights of stairs & 0.7851 & 0.2177 & 0.2789 \\
\hline $4 \mathrm{~h}$ & Climbing one flight of stair & 0.8031 & 0.0575 & 0.2114 \\
\hline $4 i$ & Bending, kneeling, or squatting & 0.6167 & 0.1355 & -0.0389 \\
\hline $4 j$ & Walking more than half a mile & 0.8121 & 0.1190 & 0.0929 \\
\hline $4 k$ & Walking a couple of hundred yards & 0.8235 & -0.0700 & 0.0380 \\
\hline 41 & Walking about one hundred yards & 0.6313 & -0.0149 & 0.0573 \\
\hline $4 \mathrm{~m}$ & Washing or dressing yourself & 0.6052 & -0.0302 & 0.1083 \\
\hline $5 a$ & Cut down the amount of time you spent on work or other activities & 0.5441 & 0.2653 & 0.3327 \\
\hline $5 b$ & Accomplished less than you would like & 0.5570 & 0.3432 & 0.3364 \\
\hline $5 c$ & Were limited in the kind of work or other activities & 0.5898 & 0.3545 & 0.3838 \\
\hline $5 d$ & Had difficulty performing the work or other activities & 0.5865 & 0.3976 & 0.3608 \\
\hline 6 & Interference with normal social activities & 0.4729 & 0.5088 & 0.4595 \\
\hline 7 & Intensity of pain around shoulder blades or in chest & 0.1619 & 0.7540 & 0.3329 \\
\hline 8 & Intensity of breathlessness & 0.5201 & 0.3874 & 0.5030 \\
\hline $9 a$ & Worried about having another pulmonary embolism? & -0.0201 & 0.2722 & 0.7017 \\
\hline $9 b$ & Felt irritable? & 0.2748 & 0.3324 & 0.7112 \\
\hline $9 c$ & Worried if having to stop anticoagulant medication? & -0.0401 & 0.1433 & 0.5288 \\
\hline $9 d$ & Became emotional more readily? & 0.2299 & 0.1613 & 0.8114 \\
\hline $9 e$ & Bothered becoming emotional more readily? & 0.2305 & 0.2645 & 0.7739 \\
\hline $9 f$ & Were depressed or in low spirits? & 0.3078 & 0.4111 & 0.5902 \\
\hline $9 \mathrm{~g}$ & Felt being a burden to family and friends? & 0.5021 & 0.3905 & 0.4279 \\
\hline $9 \mathrm{~h}$ & Were afraid to exert yourself? & 0.5391 & 0.4042 & 0.4742 \\
\hline $9 i$ & Felt limited in taking a trip? & 0.6300 & 0.3516 & 0.4126 \\
\hline $9 j$ & Were afraid of being alone? & 0.3926 & 0.1675 & 0.6115 \\
\hline
\end{tabular}

${ }^{*}$ The scree test suggests three latent factors with eigenvalues of 17.0, 3.9, and 1.7, respectively. After rotation, the three factors accounted for $35 \%$, $22 \%$, and $19 \%$ of total variance.

${ }^{\dagger}$ Numbers in bold indicate the highest factor loadings ( $\left.>0.3\right)$ for each item.

reliability. The high score correlations between the PEmbQoL and the SF-36 and the low correlation between PEmb-QoL scores and patient characteristics supported convergent and discriminant validity, respectively. Thus, the
French version of the PEmb-QoL questionnaire met standard criteria of reliability and validity for use as a patientreported measure of outcome in patients with PE, as previously shown for the Dutch version of the questionnaire. 
Table 4 Internal consistency reliability

\begin{tabular}{lllll}
\hline & PEmb-QoL questions & Number of items & Cronbach's alpha & Average inter-item correlation (r) \\
\hline Frequency of complaints & Q1 & 8 & 0.90 & 0.52 \\
Activities of daily living limitations & Q4 & 13 & 0.95 & 0.60 \\
Work-related problems & Q5 & 4 & 0.91 & 0.72 \\
Social limitations & Q6 & 1 & - & - \\
Intensity of complaints & Q7, Q8 & 2 & 0.70 & 0.54 \\
Emotional complaints & Q9 & 10 & 0.92 & 0.52 \\
PEmb-QoL summary score & Q1, Q4, Q5, Q6, Q7, Q8, Q9 & 38 & 0.92 & 0.44 \\
\hline
\end{tabular}

Only one dimension, work related problems, had a substantial ceiling effect, with $18 \%$ of patients scoring the maximum score (lowest possible quality of life) in this dimension. In contrast, a substantial floor effect was present in five out of six PEmb-QoL dimensions, i.e. more than $15 \%$ of patients had the lowest score possible, indicating the best possible quality of life. We could not exclude the possibility that the Likert scale used did not have a large enough range to accommodate the distribution of the data or that there was a social desirability bias (i.e., patients thought it made them look better if they reported high quality of life). The exceptionally high floor effect $(66 \%)$ of social limitations could be explained by the fact that this dimension consists of a single question only. Given that Klok et al. [8] already observed such floor and ceiling effects in some of the PEmb-QoL dimensions, we presumed that the floor and ceiling effects were not specifically related to the French version of the PEmb-QoL questionnaire. Indeed, floor effects $>15 \%$ were also observed in all six dimensions in the Norwegian version of the PEmb-QoL [10].

When correlating the PEmb-QoL and the SF-36 dimensions, we found a particularly strong correlation between activities of daily living limitations and physical functioning, and between work-related problems and physical role functioning. A similar observation had been made by Klok et al. [8] and might be explained by the fact that these dimensions focus on the extent of limitations when performing work or physical exercises. The relatively strong correlation between emotional

Table 5 Test-retest reliability

\begin{tabular}{ll}
\hline & $\begin{array}{l}\text { Intra-class correlation coefficient } \\
\text { (95\% confidence interval) }\end{array}$ \\
\hline Frequency of complaints & $0.94(0.92-0.96)$ \\
Activities of daily living limitations & $0.91(0.87-0.94)$ \\
Work-related problems & $0.88(0.84-0.93)$ \\
Social limitations & $0.85(0.79-0.90)$ \\
Intensity of complaints & $0.93(0.90-0.95)$ \\
Emotional complaints & $0.96(0.94-0.97)$ \\
PEmb-QoL summary score & $0.96(0.95-0.98)$ \\
\hline
\end{tabular}

complaints and social and emotional role functioning and mental health did not come as a surprise, either. The correlation between intensity of complaints and bodily pain was clinically also plausible.

The six dimensions of the PEmb-QoL were originally defined clinically and not statistically, assuming that these six dimensions would provide unique information to the treating physician [7]. The factor analysis presented by Klok et al. [8] showed that items designated to social limitations and intensity of complaints had higher loadings in other dimensions, suggesting that these two dimensions might not be justifiable. Our factor analysis supported the formation of three dimensions: limitations in daily activity (items $4 \mathrm{~b}-\mathrm{m}, 5 \mathrm{a}-\mathrm{d}$ ), symptoms (items 1a$\mathrm{h}$ and 7 ), and emotional complaints (items 9a-f and j). Notably, items 6 (Interference with normal social activities), 8 (Intensity of breathlessness), and 9 g-i (Burden to family and friends, Afraid to exert yourself, Limited in taking a trip) were not taken into account in these three dimensions because these did not contribute significantly to either of them. Given that items 6, 8, $9 \mathrm{~g}$, and 9i did not have the highest loadings in the original version of the PEmb-QoL either, a possibility would be to remove items $6,8,9 \mathrm{~g}$, and $9 \mathrm{i}$ and to replace the original six dimensions by three dimensions in the French version of the questionnaire. As an alternative, items 6, 8, 9 g-i could be grouped into the dimension on which they loaded highest, i.e. item 6 (Interference with normal social activities) in symptoms and items 8 (Intensity of breathlessness) and 9 g-i (Burden to family and friends, Afraid to exert yourself, Limited in taking a trip) in limitations in daily activity. Either way, further validations of this adapted PEmb-QoL questionnaire would be necessary. In the Norwegian version of PEmb-QoL questionnaire, an exploratory factor analysis using a different criterion to determine the number of underlying dimensions (Eigenvalues $>1$ ) resulted in six new dimensions that were not identical with the original version [10]. It is well known that measurement properties of QoL questionnaires adapted for a different population may differ from their original version due to differing translational, cultural, and methodological factors [11,21,24]. 
Table 6 Pairwise spearman correlations between SF-36 and PEmb-QoL dimension/summary score*

\begin{tabular}{|c|c|c|c|c|c|c|c|c|c|c|}
\hline PEmb-QoL & $\begin{array}{l}\text { SF-36 physical } \\
\text { functioning }\end{array}$ & $\begin{array}{l}\text { Physical role } \\
\text { functioning }\end{array}$ & $\begin{array}{l}\text { Bodily } \\
\text { pain }\end{array}$ & $\begin{array}{l}\text { General } \\
\text { health }\end{array}$ & Vitality & $\begin{array}{l}\text { Social } \\
\text { functioning }\end{array}$ & $\begin{array}{l}\text { Emotional role } \\
\text { functioning }\end{array}$ & $\begin{array}{l}\text { Mental } \\
\text { health }\end{array}$ & $\begin{array}{l}\text { Physical } \\
\text { health } \\
\text { summary }\end{array}$ & $\begin{array}{l}\text { Mental } \\
\text { health } \\
\text { summary }\end{array}$ \\
\hline $\begin{array}{l}\text { Frequency of } \\
\text { complaints }\end{array}$ & 0.47 & 0.51 & 0.68 & 0.47 & 0.60 & 0.56 & 0.49 & 0.61 & 0.49 & 0.59 \\
\hline ADL limitations & 0.90 & 0.72 & 0.61 & 0.57 & 0.54 & 0.56 & 0.64 & 0.40 & 0.80 & 0.39 \\
\hline $\begin{array}{l}\text { Work-related } \\
\text { problems }\end{array}$ & 0.62 & 0.80 & 0.66 & 0.47 & 0.58 & 0.63 & 0.59 & 0.42 & 0.70 & 0.46 \\
\hline Social limitations & 0.62 & 0.70 & 0.61 & 0.54 & 0.57 & 0.69 & 0.58 & 0.53 & 0.64 & 0.56 \\
\hline $\begin{array}{l}\text { Intensity of } \\
\text { complaints }\end{array}$ & 0.59 & 0.58 & 0.72 & 0.50 & 0.66 & 0.67 & 0.51 & 0.56 & 0.60 & 0.58 \\
\hline Emotional complaints & 0.53 & 0.68 & 0.65 & 0.55 & 0.62 & 0.83 & 0.71 & 0.71 & 0.54 & 0.76 \\
\hline $\begin{array}{l}\text { PEmb-QoL summary } \\
\text { score }\end{array}$ & 0.80 & 0.77 & 0.75 & 0.61 & 0.69 & 0.75 & 0.68 & 0.60 & 0.76 & 0.61 \\
\hline
\end{tabular}

Abbreviations: $A D L=$ Activities of daily living.

*Numbers represent Spearman correlation coefficients (r). The SF-36 summary and dimension scores were reversed for this analysis, i.e. the lower the score, the better the quality of life. All correlations between dimension/summary scores were statistically significant $(P<0.001)$.

While items 9 g-i (Burden to family and friends, Afraid to exert yourself, Limited in taking a trip) were expected to cluster in the dimension "emotional complaints", they clustered in "limitations in daily activity" in our study. A potential explanation is that the wordings "burden", "exert yourself", and "taking a trip" are not interpreted as emotional issues but rather as an obstacle to daily activity. Given that patients in our cohort were older (63 vs. 56 years) and less likely to have cardiopulmonary comorbidity (13\% vs. $20 \%)$ and obesity (13\% vs. $39 \%)$ than patients in the study by Klok et al. [8], we could not exclude the possibility that these differences in patient baseline characteristics did not contribute to differences in self-reported health measures.

To facilitate the comparison of PE-related quality of life across studies, we created an overall PEmb-QoL summary score using all items, except Q2 ("At what time of day are your lung symptoms most intense?") and Q3 ("Compared to one year ago, how would you rate the condition of your lungs in general now?"), which were never scored. This overall score met standard criteria of acceptability, reliability, reproducibility, and validity for use as a summary patient-reported measure of outcome in patients with acute PE.

Our study has potential limitations. First, only $42 \%$ of potentially eligible persons with PE completed the questionnaires, mostly, because they were unreachable or refused to participate. Thus, we could not entirely exclude the possibility that elderly and sicker patients were underrepresented in our study. However, our enrolment rate compared well with previous studies, in which less than $40 \%$ of screened patients with VTE underwent quality of life assessments [10,25]. Second, we could not ascertain the number of missing items per patient because missing items were completed by participants following contact by phone with a study collaborator. However, less than a third of patients had to be contacted because they had one or more missing items. Finally, we were not able to assess responsiveness of the PEmb-QoL questionnaire, that is, its ability to detect a clinically meaningful change over time [8].

In conclusion, despite the presence of some floor and ceiling effects, the French version of the PEmb-QoL questionnaire meets standard criteria of reliability and

Table 7 Pairwise spearman correlations between PEmb-QoL dimensions/summary score and baseline characteristics*

\begin{tabular}{|c|c|c|c|c|c|}
\hline & Age & Female gender & Obesity & Cancer & Cardiopulmonary comorbidity ${ }^{\ddagger}$ \\
\hline Frequency of complaints & -0.05 & 0.07 & $0.21 \dagger$ & -0.15 & 0.03 \\
\hline ADL limitations & $0.32+$ & -0.08 & 0.18 & -0.07 & 0.12 \\
\hline Work-related problems & 0.18 & -0.16 & -0.02 & -0.05 & 0.17 \\
\hline Social limitations & 0.02 & 0.01 & $0.21+$ & -0.12 & 0.11 \\
\hline Intensity of complaints & -0.01 & -0.06 & 0.14 & -0.16 & 0.10 \\
\hline Emotional complaints & -0.10 & -0.07 & 0.13 & -0.18 & 0.13 \\
\hline Overall PEmb-QoL summary score & 0.12 & -0.07 & 0.16 & -0.15 & 0.12 \\
\hline
\end{tabular}

Abbreviations: $A D L=$ Activities of daily living.

*Numbers represent Spearman correlation coefficients $(r)$.

${ }^{\dagger}$ Correlation with statistical significance $(P<0.05)$.

${ }^{\ddagger}$ Any cardiac disease with systolic or diastolic ventricular dysfunction or any obstructive or restrictive pulmonary disease. 
validity for use as a patient-reported measure of quality of life and symptoms in patients with PE. Thus, the French version of the PEmb-QoL can be used with confidence in prospective studies to assess PE-specific quality of life and symptoms.

\section{Additional file}

Additional file 1: The French version of the PEmb-QoL questionnaire.

\section{Competing interests}

The authors declare that they have no competing interests.

\section{Authors' contributions}

$M R, M M, A L, O H, D A$ participated in the conception of the study and its design, carried out the data analyses and drafting of the manuscript. MR and MM collected the data. OH, FAK, and DMC critically revised the manuscript. All authors read and approved the final manuscript.

\section{Author details}

'Department of Internal Medicine, Lausanne University Hospital, CHUV, Rue du Bugnon 46, 1011 Lausanne, Switzerland. ²Division of General Internal Medicine, Bern University Hospital, Bern, Switzerland. ${ }^{3}$ Department of Clinical Research, and Institute of Social and Preventive Medicine (ISPM), CTU Bern, University of Bern, Bern, Switzerland. Emergency Department, Lausanne University Hospital, Lausanne, Switzerland. ${ }^{5}$ Department of Thrombosis and Hemostasis, Leiden University Medical Center, Leiden, the Netherlands. ${ }^{6}$ Department of Vascular Medicine, Academic Medical Center, Amsterdam, the Netherlands.

Received: 18 April 2014 Accepted: 15 November 2014

Published online: 03 December 2014

\section{References}

1. Konstantinides S: Acute pulmonary embolism. N Engl J Med 2008, 359:2804-2813.

2. Rosendaal FR, Van Hylckama Vlieg A, Doggen CJM: Venous thrombosis in the elderly. J Thromb Haemost 2007, 5(Suppl 1):310-317.

3. Kahn SR, Ginsberg JS: Relationship between deep venous thrombosis and the postthrombotic syndrome. Arch Intern Med 2004, 164:17-26.

4. Spencer FA, Gore JM, Lessard D, Douketis JD, Emery C, Goldberg RJ: Patient outcomes after deep vein thrombosis and pulmonary embolism: the Worcester Venous Thromboembolism Study. Arch Intern Med 2008, 168:425-430.

5. Pengo $\mathrm{V}$, Lensing AW, Prins MH, Marchiori A, Davidson BL, Tiozzo F, Albanese P, Biasiolo A, Pegoraro C, lliceto S, Prandoni P, Thromboembolic Pulmonary Hypertension Study Group: Incidence of chronic thromboembolic pulmonary hypertension after pulmonary embolism. N Engl J Med 2004, 350:2257-2264.

6. Kahn SR, Lamping DL, Ducruet T, Arsenault L, Miron MJ, Roussin A, Desmarais S, Joyal F, Kassis J, Solymoss S, Desjardins L, Johri M, Shrier I, VETO Study investigators: VEINES-QOL/Sym questionnaire was a reliable and valid disease-specific quality of life measure for deep venous thrombosis. J Clin Epidemiol 2006, 59:1049-1056.

7. Cohn DM, Nelis EA, Busweiler LA, Kaptein AA, Middeldorp S: Quality of life after pulmonary embolism: the development of the PEmb-QoL questionnaire. J Thromb Haemost 2009, 7:1044-1046.

8. Klok FA, Cohn DM, Middeldorp S, Scharloo M, Buller HR, van Kralingen KW, Kaptein AA, Huisman MV: Quality of life after pulmonary embolism: validation of the PEmb-QoL Questionnaire. J Thromb Haemost 2010, 8:523-532.

9. van Es J, den Exter PL, Kaptein AA, Andela CD, Erkens PM, Klok FA, Douma RA Mos IC, Cohn DM, Kamphuisen PW, Huisman MV, Middeldorp S: Quality of life after pulmonary embolism as assessed with SF-36 and PEmb-QoL. Thromb Res 2013, 132:500-505.

10. Tavoly M, Jelsness-Jorgensen LP, Wik HS, Roaldsnes C, Sandset PM, Ghanima W: Quality of life after pulmonary embolism: first cross-cultural evaluation of the pulmonary embolism quality-of-life (PEmb-QoL) questionnaire in a Norwegian cohort. Qual Life Res 2014, ahead of print
11. Beaton DE, Bombardier C, Guillemin F, Ferraz MB: Guidelines for the process of cross-cultural adaptation of self-report measures. Spine (Phila Pa 1976) 2000, 25:3186-3191.

12. PIOPED The Investigators: Value of the ventilation/perfusion scan in acute pulmonary embolism: results of the prospective investigation of pulmonary embolism diagnosis (PIOPED). JAMA 1990, 263:2753-2759.

13. Remy-Jardin M, Remy J, Wattinne L, Giraud F: Central pulmonary thromboembolism: diagnosis with spiral volumetric CT with the single-breath-hold technique-comparison with pulmonary angiography. Radiology 1992, 185:381-387.

14. Terwee CB, Bot SD, de Boer MR, van der Windt DA, Knol DL, Dekker J, Bouter LM, de Vet HC: Quality criteria were proposed for measurement properties of health status questionnaires. J Clin Epidemiol 2007, 60:34-42.

15. Altman DG: Practical statistics for medical research. London: Chapman and Hall; 1991

16. Lamping DL, Schroter S, Kurz X, Kahn SR, Abenhaim L: Evaluation of outcomes in chronic venous disorders of the leg: development of a scientifically rigorous, patient-reported measure of symptoms and quality of life. J Vasc Surg 2003, 37:410-419.

17. Leplège A, Ecosse E, Verdier A, Perneger TV: The French SF-36 health survey: translation, cultural adaptation and preliminary psychometric evaluation. J Clin Epidemiol 1998, 51:1013-1023.

18. Ware JE Jr, Sherbourne CD: The MOS 36-item short-form health survey (SF-36). I. conceptual framework and item selection. Med Care 1992, 30:473-483

19. Ware JE Jr, Kosinski M, Dewey JE: How to score - Version 2 of the SF-36 Health Survey (Standard and Acute Forms). Lincoln, Rhode Island: QualityMetric, Incorporated; 2001

20. Ware JE Jr: SF-36 health survey update. Spine (Phila Pa 1976) 2000, 25:3130-3139.

21. de Vet HC, Ader HJ, Terwee CB, Pouwer F: Are factor analytical techniques used appropriately in the validation of health status questionnaires? a systematic review on the quality of factor analysis of the SF-36. Qual Life Res 2005, 14:1203-1218.

22. Cattell RB: The scree test for the number of factors. Multivariate Behav Res 1966, 1:245-276.

23. Nunnally JC, Bernstein $\mathrm{H}$ : Psychometric theory. 3rd edition. New York: McGraw-Hill; 1994.

24. Aaronson N, Alonso J, Burnam A, Lohr KN, Patrick DL, Perrin E, Stein REK Assessing health status and quality-of-life instruments: attributes and review criteria. Qual Life Res 2002, 11:193-205

25. Kahn SR, Shbaklo H, Lamping DL, Holcroft CA, Shrier I, Miron MJ, Roussin A, Desmarais S, Joyal F, Kassis J, Solymoss S, Desjardins L, Johri M, Ginsberg JS: Determinants of health-related quality of life during the 2 years following deep vein thrombosis. J Thromb Haemost 2008, 6:1105-1112.

\section{doi:10.1186/s12955-014-0174-4}

Cite this article as: Rochat et al:: Quality of life after pulmonary embolism: validation of the French version of the PEmb-QoL questionnaire. Health and Quality of Life Outcomes 2014 12:174.

\section{Submit your next manuscript to BioMed Central and take full advantage of:}

- Convenient online submission

- Thorough peer review

- No space constraints or color figure charges

- Immediate publication on acceptance

- Inclusion in PubMed, CAS, Scopus and Google Scholar

- Research which is freely available for redistribution 